\title{
LA FOULE RELIGIEUSE DE LOURDES CHEZ ZOLA ET HUYSMANS
}

\section{Eduardo Cintra Torres}

Société d'études soréliennes | "Mil neuf cent. Revue d'histoire intellectuelle »

2010/1 n $28 \mid$ pages 35 à 58

ISSN 1146-1225

ISBN 2912338280

Article disponible en ligne à l'adresse :

https://www.cairn.info/revue-mil-neuf-cent-2010-1-page-35.htm

Distribution électronique Cairn.info pour Société d'études soréliennes.

(C) Société d'études soréliennes. Tous droits réservés pour tous pays.

La reproduction ou représentation de cet article, notamment par photocopie, n'est autorisée que dans les limites des conditions générales d'utilisation du site ou, le cas échéant, des conditions générales de la licence souscrite par votre établissement. Toute autre reproduction ou représentation, en tout ou partie, sous quelque forme et de quelque manière que ce soit, est interdite sauf accord préalable et écrit de l'éditeur, en dehors des cas prévus par la législation en vigueur en France. Il est précisé que son stockage dans une base de données est également interdit. 


\title{
La foule religieuse de Lourdes chez Zola et Huysmans*
}

\author{
Eduardo Cintra Torres
}

\section{Étudier la foule vers 1894}

Lorsque Émile Zola publie son roman sur Lourdes en 1894, le concept de foule est quasiment un synonyme de «foule criminelle» - c'était d'ailleurs un thème de criminologues. Le délégué portugais au Congrès d'anthropologie criminelle de Bruxelles (août 1892), où Gabriel Tarde a présenté son premier travail sur le sujet - une communication intitulée «Les crimes des foules» -, voit dans la foule un «être animé» soudé en «un même corps» dans lequel «réapparaissent les instincts sauvages primitifs »: «La foule ainsi agitée est un organisme social rétrograde, capable des iniquités les plus atroces ${ }^{1} »$. L'auteur cite Tarde et Scipio Sighele à propos des crimes collectifs et reconnaît «qu'il y a eu et qu'il y aura toujours des associations » qui ont «pour objectif la satisfaction d'exigences morales telles que les associations religieuses ou

* Traduction écourtée de l'article paru dans Análise social, XLII, 184, 2007, p. 733755, revue de l'Institut de sciences sociales de l'Université de Lisbonne (ICS-UL). Abréviations utilisées: $\mathbf{H}$ = Joris-Karl Huysmans, Les foules de Lourdes (1906), préf. de François Angelier, Grenoble, Jérôme Million, 1993; RE = Émile Zola, Le roman expérimental, Paris, Charpentier Éd., 1880, 2e éd.; G = Id., Germinal (1885), Henri Mitterand (ed.), préf. d'André Wurmser, Paris, Gallimard, 1978; L = Id., Lourdes (1894), Jacques Noiray (ed.), Paris, Gallimard, 1995 ; CE = Id., Euvres complètes, VII, Henri Mitterand (ed.), préf. de Dominique Fernandez et introd. de René Ternois, Paris, Fasquelle, 1968.

1. Manuel Ferreira-Deusdado, A anthropologia criminal e o Congresso de Bruxelas, Lisbonne, Imprensa Nacional, 1894, p. 80-81. 
scientifiques ${ }^{2}$ ", mais il ne fait aucune référence aux agrégats à caractère religieux ayant la forme d'une foule et des finalités édifiantes.

À l'instar du rapporteur portugais, les auteurs ayant étudié la foule à l'époque de la publication de Lourdes étaient davantage préoccupés par les foules prolétaires et urbaines, qui envahissaient de plus en plus l'espace public et qu'on assimilait aux «classes dangereuses».

Hippolyte Taine, Scipio Sighele, Gabriel Tarde, Gustave Le Bon et Henry Fournial, «tous semblent être parvenus, de façon indépendante, aux mêmes conclusions: les foules sont normalement très émotives et irrationnelles, plus destructives que constructives, ayant besoin d'une autorité forte ${ }^{3}$ ». Un tel constat ne découlait pas seulement d'un positionnement politique commun, car si la plupart de ces auteurs étaient bien des libéraux, d'autres comme Cesare Lombroso et Enrico Ferri arrivaient à des conclusions très proches alors qu'ils étaient membres fondateurs du Parti socialiste italien. Quant à Sighele, le premier à avoir consacré une monographie à la foule, il prônait un «socialisme d'amour» contre un «socialisme de rancœur». Mais tous regardaient la foule, avec un respect mâtiné de crainte, en tant qu'objet empirique qui se présentait à eux dans la rue, et dans la littérature où ils puisaient leur source ${ }^{4}$.

C'est pourquoi ni La foule criminelle de Sighele (1891), ni le premier texte de Tarde sur la foule, «Les foules et les sectes criminelles» $(1893)^{5}$, n'accordent d'importance aux foules religieuses. Dans l'étude des crimes des foules, Sighele envisage difficilement l'émergence de collectifs généreux et positifs: «La foule est un terrain où le microbe du mal se développe très facilement, tandis que le microbe du bien meurt presque toujours ${ }^{6} . »$ Ce n'est qu'à la $3^{\mathrm{e}}$ édition de La foule criminelle, en 1903, quasiment une décennie après Lourdes, que Sighele ajoute une seconde partie consacrée à «l'intelligence de la foule », dans laquelle il met en avant des aspects positifs du comportement collectif ${ }^{7}$.

Aussi bien dans la Psychologie des foules de Le Bon (1895) que dans le texte de Tarde «Le public et la foule» $(1898)^{8}$, on voit cependant

\section{Ibidem.}

3. Jaap Van Ginneken, Crowds, psychology and politics: 1871-1899 (1989), Cambridge, Cambridge University Press, 1992, p. 6.

4. Ibid., p. 16-17, 75 .

5. Repris dans Gabriel Tarde, L'opinion et la foule (1901), Paris, Puf, 1989.

6. Cité par Susanna Barrows, Miroirs déformants. Réflexions sur la foule en France à la fin du XIX siècle (1981), s.1., Aubier, 1990, p. 115.

7. J. Van Ginneken, op. cit., p. 99.

8. Repris dans G. Tarde, op. cit. 
apparaître la figure de la foule pacifique et conservatrice, et le postulat de la capacité du meneur à conduire la foule. On aurait fort à parier que l'attention vis-à-vis de la foule d'un nouveau genre, comme celle religieuse de Lourdes - abordée pour la première fois et en profondeur dans le populaire roman de Zola -, a procuré aux deux auteurs une vue plus large de la foule, moins restreinte au cliché d'une foule populaire ou prolétaire dangereuse.

Cette période agitée, ayant vu apparaître à partir de 1890 les premières et grandes manifestations du $1^{\mathrm{er}}$ mai, est aussi marquée par l'épisode boulangiste (1886-1889), qui a montré la capacité d'un leader à mobiliser de grandes foules de façon démagogique ${ }^{9}$. Certains observateurs postulent dès lors que les classes travailleuses recherchent, instinctivement, un chef; Jules Ferry déclare en 1889 que le peuple de Paris peut être dupé à l'instar «des foules crédules qui [...] ont imposé les supercheries de Lourdes comme miracles authentiques ${ }^{10}$ ». C'est aussi l'époque où est publiée l'encyclique de Léon XIII Rerum novarum (1891). Ce texte, qui proposait une collaboration entre l'État, l'Église, les patrons et les travailleurs, considérait ces derniers comme les victimes d'abus fréquents, nuançant de la sorte la vision implacable de «classes dangereuses». C'est encore le moment de l'Exposition universelle de Paris qui, en 1889, accueille d'énormes foules pacifiques: trente-trois millions de visiteurs. En dépit de ces manifestations de la diversité du phénomène collectif, les premiers théoriciens de la foule se concentrent sur le nouveau stéréotype de la foule criminelle des travailleurs.

Le texte de Tarde de 1893 fait référence aux foules de Lourdes, mais c'est pour mettre en relief sa thèse à propos des foules criminelles et du pouvoir de la foule vis-à-vis de ceux qui l'intègrent: il est alors question d'imitation, de suggestion et d'interaction sociale. Il est tout à fait remarquable que, malgré le jugement porté sur les «foules ferventes» de Lourdes - «ces foules pieuses n'ont rien de féroce»-, Tarde n'y fasse allusion que dans le but de faire ressortir le phénomène de l'imitation au sein des "foules criminelles", son principal sujet ${ }^{11}$.

Nous ignorons si le roman de Zola a directement influencé les réflexions de Tarde, de Le Bon et d'autres auteurs qui revendiquent une écriture scientifique sur les foules. Mais, compte tenu de la puissante

9. S. Barrows, op. cit.; J. Van Ginneken, op. cit.

10. Cité par J. Van Ginneken, ibid., p. 158.

11. G. Tarde, op. cit., p. 182-183. 
influence de Germinal sur l'ensemble d'entre eux, qu'ils proviennent de l'école italienne ou française, il est tout à fait légitime d'émettre l'hypothèse que le roman de Zola ait aussi aiguillé leurs analyses. À l'époque, la littérature a compris avant la science l'importance de certains phénomènes collectifs ${ }^{12}$. Zola est cité par Sighele, Le Bon et bien d'autres théoriciens. Il est cité jusque dans les prétoires. En 1887, Giuseppe Pugliese, qui publie la même année un ouvrage sur le Délit collectif, défend à Bari des prévenus accusés du délit d'association en «foule criminelle» en ayant recours, en guise d'explication, aux narrations de révoltes fictives contenues dans les œuvres romanesques de Zola, Alessandro Manzoni et Victor Hugo ${ }^{13}$.

Dans un texte postérieur, Sighele, grand admirateur de Zola, fait référence à Lourdes comme une manifestation de plus de «l'âme collective» de la foule dans l'œuvre du romancier: «De Germinal à La débâcle et à Lourdes, où les protagonistes individuels sont entourés par des foules vivantes et frémissantes d'ouvriers, de soldats et de mystiques, foules différentes par leurs compositions et leurs buts, mais qui toutes mettent en scène le problème épouvantablement obscur de l'âme collective ${ }^{14}$.»

Un an après Lourdes, Le Bon ne fait aucune référence à la possibilité pour la religion de se manifester à travers la foule. Il préfère insister sur la «destruction des croyances religieuses »: «Les foules ne veulent plus aujourd'hui des dieux dont [leurs anciens maîtres] ne voulaient pas hier et qu'ils ont contribué à briser ${ }^{15}$.» Ce qu'il perçoit est autre chose, ce sont les «formes religieuses que revêtent toutes les convictions des foules». "Aussi - affirme-t-il dans son style aphoristique est-ce une bien inutile banalité de répéter qu'il faut une religion aux foules, puisque toutes les croyances politiques, divines et sociales ne s'établissent chez elles qu'à la condition de revêtir toujours la forme religieuse, qui les met à l'abri de la discussion ${ }^{16}$.» En d'autres termes, Le Bon n'écrit pas sur les foules religieuses car cela lui semble insignifiant, compte tenu qu'à leur manière, elles sont toutes religieuses.

En ce qui concerne Tarde, la réflexion sur les foules religieuses, qu'il livre dans son texte de 1898, «Le public et la foule», lui permet d'établir quelques discriminations: «Nous ne confondrons pas avec

12. Serge Moscovici, L'âge des foules, Paris, Fayard, 1981, p. 119 n.

13. J. Van Ginneken, op. cit., p. 71.

14. Scipio Sighele, Littérature et criminalité, Paris, Giard et Brière, 1908, p. 127.

15. Gustave Le Bon, Psychologie des foules (1895), Paris, Puf, 1998, p. 1, 3.

16. Ibid., p. 39, 41. 
les foules orantes et processionnelles que des membres du clergé conduisent à Lourdes, les foules révolutionnaires et hurlantes soulevées par un jacobin, ou les foules pitoyables et affamées de grévistes menées par un syndicat ${ }^{17}$ ". Dès lors, il peut franchir un pas supplémentaire vers une typologie moins idéologique, davantage sociologique, des «quatre manières d'être» des foules: les «expectantes", les «attentives», les «manifestantes» et les «agissantes ${ }^{18}$ ». Par rapport à sa première prise de position, cinq ans auparavant, il révise son jugement quant aux foules positives:

On peut distinguer les foules d'action en foules d'amour et foules de haine. [...] il y a une variété des foules d'amour, très répandue, qui joue un rôle social des plus nécessaires et des plus salutaires, et sert de contrepoids à tout le mal accompli par toutes les autres espèces de rassemblements. Je veux parler de la foule de fête, de la foule de joie, de la foule amoureuse d'elle-même, ivre uniquement du plaisir de se rassembler pour se rassembler. [...] En somme les foules sont loin de mériter dans leur ensemble le mal qu'on en a dit et quej'en ai pu dire moi-même à l'occasion. Si l'on met en balance l'curre quotidienne et universelle des foules d'amour, surtout des foules de fêtes, avec l'ceuvre intermittente et localisée des foules de haine, on devra reconnaître en toute impartialité, que les premières ont beaucoup plus contribué à tisser ou resserrer les liens sociaux que les secondes à déchirer par endroits ce tissu ${ }^{19}$.

L'évocation des foules festives est significative car elle peut rappeler celles de Lourdes qui sont aussi des foules empreintes de révérence et de bonheur ${ }^{20}$, «hautement expressives, extatiques et joyeuses», de sorte qu'il est possible d'étudier l'expérience «des pèlerinages, des miracles et des apparitions dans des contextes de foule ${ }^{21}$ ».

En quelque sorte, le Zola de 1894 est donc responsable de l'inclusion de la foule religieuse et festive dans le cadre de la théorie sur les comportements collectifs. Comme nous le verrons, son roman était aussi novateur sur un autre point: il admettait que les « congrégations

17. G. Tarde, op. cit., p. 22.

18. Ibid., p. 23-26.

19. Ibid., p. 26-27.

20. Voir John Lofland, «Crowd joys», Urban Life, X, 4, janvier 1982, p. 355-381.

21. Phillip Davis, Jacqueline Boles, «Pilgrim apparition work: Symbolization and crowd interaction when the virgin mary appeared in Georgia ", Journal of Contemporary Ethnography, XXXII, 4, août 2003, p. 375. 
marquées par leur révérence» pouvaient être manipulées par leurs conducteurs ou meneurs. L'existence de foules menées par la religion, comme à Lourdes, ou par un leader populiste, comme Boulanger, a permis à Le Bon de recommander aux élites d'apprendre à dominer les foules, préfigurant de la sorte les méthodes mises en œuvre (meetings, etc.) par les partis de Mussolini et d'Hitler ${ }^{22}$.

Dès 1895, les auteurs qui étudient la «foule» sur le mode scientifique élargissent donc ce concept au-delà des rassemblements des «classes dangereuses», incorporant les «foules d'amour» ou, comme avec Le Bon, envisageant la possibilité qu'en démocratie on puisse orienter la foule vers des objectifs politiques contraires à ceux du socialisme.

\section{Zola et le phénomène de Lourdes}

En 1894, alors qu'il achève le cycle des Rougon-Macquart, Zola publie le roman Lourdes, un énorme succès éditorial. Le romancier prétend illustrer dans ce roman à thèse comment les phénomènes de la foi survivent et renaissent dans le siècle de la raison et de la science. Il a été le premier auteur de renom à se pencher sur le phénomène de la dévotion des masses à Lourdes. Dans cette commune rurale des Pyrénées, la jeune Bernadette Soubirous (1844-1879) avait affirmé, en 1858, avoir vu et entendu dix-huit fois la vierge Marie, de qui elle prétendait avoir reçu des instructions. La foule pèlerine était à l'origine du phénomène, Bernadette ayant fait référence à un appel de la Vierge aux foules: elle avait demandé que «les peuples s'y rendissent en procession, de tous les points de la terre» (L: 129). La commune était devenue un lieu de pèlerinage. Par la suite, l'Église reconnut les apparitions.

Selon Zola, ce phénomène est différent des dévotions miraculeuses médiévales ou modernes - non pas par la forme, similaire à d'autres apparitions de la vierge Marie à de jeunes bergers inventoriées depuis des siècles, mais par l'époque et la société dans lesquelles il naît. Primo, le miracle a lieu au milieu d'un siècle dit de progrès et de lumière. Secundo, il se produit dans un pays cosmopolite et urbain. Tertio, le phénomène attire à Lourdes d'innombrables trains spéciaux

22. J. Lofland, art. cit., p. 371; Stephen Reicher, «"The crowd” Century: Reconciling practical success with theoretical failure », British Journal of Social Psychology, 35, 1996, p. 535-553. 
avec des centaines de milliers, voire des millions de pèlerins venus de toute la France et d'Europe - et même depuis les contrées les plus reculées comme Macao, territoire cité aussi bien dans le roman de Zola (L: 422) que dans le récit de Huysmans (H:147). De 1878 à 1903, Lourdes est devenue une destination de masse: la commune accueille 3500 pèlerinages nationaux et 3200000 fidèles (Noiray, in $\mathrm{L}: 11)$.

\section{Le roman comme document et son intention théorisante}

Avant d'entrer dans le vif du sujet - les foules de Lourdes dans une œuvre littéraire -, nous nous devons de signaler la valeur du roman de Zola en tant que document. Il s'agit là d'un aspect essentiel, étant donné que la fonction documentaire des œuvres littéraires naturalistes a permis aux érudits non romanciers d'en faire usage comme sources légitimes pour l'étude de la foule. Nous faisons référence à des romans, des contes ou des feuilletons de Victor Hugo, Gabriele D’Annunzio, Alessandro Manzoni, Gustave Flaubert, Émile Zola, Edgar Allan Poe, Emily Brontë, Charles Dickens, George Eliot ou Walter Scott, auxquels nous ajouterons le récit historique biaisé d'Hippolyte Taine sur la période de la Révolution française, «un récit "scientifique" " qui, lui aussi, «reposait sur la liberté littéraire» d'auteurs comme ceux que nous venons de $\operatorname{citer}^{23}$.

Taine fut l'un des maîtres de la fusion des textes scientifique et littéraire et "peu de lecteurs de Taine ont apprécié autant que Zola sa manière de fondre littérature et science ${ }^{24} »$. Les deux auteurs procédaient de même, mais Taine est devenu suspect parce qu'il écrivait au nom de la science, alors que Zola situait la démarche de Taine «dans son véritable domaine: le roman ${ }^{25}$ ». Le naturalisme littéraire encourageait les théoriciens à citer des réalités transfigurées par la fiction, d'autant plus que ceux-ci n'avaient ni les moyens ni l'initiative d'étudier les foules «de l'intérieur». "Tous ceux qui ont écrit sur la foule, de Taine à Le Bon en passant par Zola, ont estimé faire une œuvre scientifique», affirme Barrows. "Tous se sont réclamés de l'histoire et tous ont mélangé le réel et l'imaginaire. Mais seul Zola en avait le droit», parce que c'était un homme de fiction ${ }^{26}$.

23. J. Van Ginneken, op. cit., p. 21.

24. S. Barrows, op. cit., p. 86.

25. Ibid., p. 97.

26. Ibid., p. 175. 
Par leur méthode d'enquête, les naturalistes comme Zola parvenaient à être plus proches de leur objet que les théoriciens issus de la criminologie ou de la science politique. Il n'est donc guère surprenant que «l'approche sociologique ou pré-sociologique» soit "plus élaborée chez les écrivains", note un auteur ayant comparé les romans hostiles à la Commune de Paris et les théories contemporaines sur la foule ${ }^{27}$.

La démarche d'enquête de Zola s'ajustait à l'intention naturaliste de son art. Zola a visité Lourdes en 1892 et plus longuement en 1893. Il s'y est entretenu avec des personnages importants et il a lu des ouvrages sur les aspects religieux. Nous pouvons dire que son travail de recherche, si ce ne fut celui d'un ethnologue, a été celui d'un reporter d'investigation: "Je dis ce que je vois, je verbalise simplement» $(\mathrm{G}: 12)$. Le roman reproduit en détail les innombrables annotations prises à Lourdes ("Mon Voyage à Lourdes", in CE: 401504), notamment les descriptions des foules et même des détails comme les ex-roto qu'il a vus dans une église (L: 428; CE: 458). Tous les protagonistes et autres principaux personnages du roman ont un lien avec des personnes réelles que Zola a connues ou sur lesquelles il a recueilli des informations (Mitterand, in E: 474).

On peut dire de Lourdes ce que Wurmser affirme de l'ensemble de son œuvre: Zola «romance des reportages». C'est pourquoi ce roman peut servir comme document pour connaître le Lourdes de la fin du $\mathrm{XIX}^{\mathrm{e}}$ siècle sous plusieurs traits: religieux, social, psychique et même politique. La "précision du reportage» et «une véritable réussite dans l'intégration naturelle de la chose vue à la trame romanesque» donnent à Lourdes « son caractère irremplaçable de document ». Zola a atteint ici, mieux que dans tout autre œuvre, "l'idéal naturaliste de la transparence» (Wurmser, in G: 7, 11, 14). Même si Lourdes révèle l'intérêt renouvelé de Zola pour la force individuelle et sociale $\mathrm{du}$ «rêve», ce roman participe du «roman expérimental» ou d'une «littérature déterminée par la science».

C'est ainsi-affirmait Zola, à propos des romanciers naturalistes au nombre desquels il se comptait - que nous faisons de la sociologie pratique et que notre besogne aide aux sciences politiques et économiques. [...] Être maître du bien et du mal, régler la vie,

27. Dominique Cochart, «Les foules et la Commune. Analyse des premiers écrits de psychologie des foules", Recherches de psychologie sociale, 4, 1982, p. 56. 
régler la société, résoudre à la longue tous les problèmes du socialisme, apporter surtout des bases solides à la justice en résolvant par l'expérience les questions de criminalité, n'est-ce pas là être les ouvriers les plus utiles et les plus moraux du travail humain? (RE: 24.)

Zola souhaitait appliquer à la lettre la méthode expérimentale proposée par Claude Bernard. En partant de l'observation, le romancier faisait une expérience à travers la création de personnages et d'une action déterminée par le milieu et l'hérédité. À tel point que, comme l'homme de science, il mettait de côté sa propre sensibilité:

Le romancier expérimentateur est donc celui qui accepte les faits prouvés, qui montre dans l'homme et dans la société le mécanisme des phénomènes dont la science est maîtresse, et qui ne fait intervenir son sentiment personnel que dans les phénomènes dont le déterminisme n'est point encore fixé, en tâchant de contrôler le plus quil le pourra ce sentiment personnel, cette idée a priori, par l'observation et par l'expérience (RE: 52).

La soumission du romancier à la méthode expérimentale le conduit à une attitude ambivalente face à la théorie. D'une part, l'expérimentateur se doit d'être méfiant et mettre de côté toutes les théories - de façon qu'elles n'empêchent pas la «vérité» de se manifester à partir de l'expérience. D'autre part, du fait que la méthode expérimentale renvoie vers plusieurs directions, il se doit de faire usage de théories à la carte: «nous ne pouvons toujours échapper à ce besoin de notre intelligence» qui «nous porte à vouloir connaître l'essence des choses", c'est pourquoi «il faut alors accepter le système philosophique qui s'adapte le mieux à l'état actuel des sciences, mais simplement à un point de vue spéculatif». "Toutes les théories - remarque Zola - sont admises, et la théorie qui l'emporte est celle qui explique le plus de choses.» (RE: 44, 43.) D'ailleurs, comment pourrait-il rejeter la théorie s'il attribue au roman un désir de vérité et de morale?

Le roman naturaliste est une littérature à thèse qui veut rendre le monde meilleur. Aussi y a-t-il dans Lourdes, quoique parfois d'une façon confuse, une intention théorisante qui toutefois reste dissimulée quant à ses visées, car «un expérimentateur n'a pas à conclure»: «L'expérience conclut pour lui. [...] Comme nous sommes des expérimentateurs sans être des praticiens, nous devons nous contenter de chercher le déterminisme des phénomènes sociaux, en laissant aux 
législateurs, aux hommes d'application, le soin de diriger tôt ou tard ces phénomènes.» (RE:29.) En d'autres termes, il incombe à d'autres de tirer des leçons théoriques de l'expérimentalisme littéraire.

\section{Zola, précurseur de l'étude de la foule amoureuse}

Un des éléments fondamentaux de ce roman tient à la présence écrasante de la foule dans le sanctuaire. En septembre 1892, lors de la première visite au sanctuaire, Zola rédige une note pour « un roman sur Lourdes», dans laquelle il fait allusion aux «foules accourues, cent cinquante mille par an, je crois", se proposant de "retrouver, étudier et peindre ce duel incessant entre la science et le besoin du surnaturel». Il imagine une intrigue avec des personnages tels que "des prêtres, des évêques, des croyants et des spéculateurs, des foules surtout», car les "grandes foules» sont bien son centre d'intérêt (cité par Mitterand, in E: 473). Zola envisage alors d'écrire un livre simple, "uniquement des malades, leur souffrance et leurs appels. Rien d'autre, ni amours ni intrigues secondaires» (cité par Ternois, in $\mathrm{E}: 19)$. Bref, un livre entièrement consacré à la foule.

Une fois de plus, la foule est pour Zola un personnage de premier plan. Bien qu'elle fût déjà protagoniste dans Germinal, suscitant un débat sur la «foule criminelle» des prolétaires - sans moyens de se manifester pour leurs droits -, le romancier a également écrit des scènes impressionnantes dans La fortune des Rougon, Nana, et $A u$ bonheur des dames, romans eux aussi antérieurs à la théorisation sur la foule ${ }^{28}$. Mais, grâce à l'art prodigieux avec lequel Zola traite des foules, on peut dire que «les foules de Lourdes sont aussi puissantes que celles de Germinal ${ }^{29}{ }^{\text {». }}$

28. J.H. Matthews, «Émile Zola and Gustave Le Bon», Modern Language Notes, LXXIII, 2, février 1958, p. 109-113. Matthews commet une erreur chronologique lorsqu'il exclut Lourdes des ouvrages de Zola faisant référence à la foule déjà publiés lors de la sortie du livre de Le Bon (1895). Lourdes est sorti un an avant la Psychologie des foules; la presse en parlait depuis 1892. Dès 1891, Zola avait fait savoir son intention d'écrire sur Lourdes, comme il est indiqué dans le Journal des frères Goncourt: dans un salon littéraire, il affirme avoir été «frappé, stupéfié par le spectacle de ce monde de croyants hallucinés [de Lourdes] et qu'il y aurait de belles choses à écrire sur ce renouveau de la foi» (cité par Mitterand, «Notice», in $\mathrm{E}:$ : 472). Matthews signale que Zola «n'a ni correspondu avec Le Bon, ni reçu du courrier de sa part. Il n'a pas cité Le Bon, mais sa présentation dramatique a anticipé la monographie de celui-ci, ce qui illustre la compréhension authentique et instinctive du romancier à propos de la psychologie de la foule» (J.H. Matthews, art. cit., p. 113).

29. Henri Mitterand, entretien, l'Humanité, 30 septembre 2002. 


\section{La foule comme solution, non comme problème}

Lourdes débute par le voyage à bord d'un des trains spéciaux ParisLourdes et s'achève par le voyage retour. Cette structure narrative correspond à l'expérience même des pèlerins et permet au romancier de montrer qu'une "fraternité extraordinaire les unissait» le temps du pèlerinage. L'expérience, proprement liminale - au sens de Van Gennep -, est saisissante et domine les sensations et l'esprit tant qu'elle dure. Au retour, le pèlerinage est défini comme l'expérience d'un «rêve prodigieux» (L: 267, 578).

Les protagonistes de Zola sont Pierre Froment, un prêtre incroyant, et Marie, son amie et amour d'enfance, paralytique depuis le début de sa jeunesse. Lui va à Lourdes en quête de foi; quant à elle, par la foi, elle aspire au miracle. La guérison s'opère, mais Zola l'attribue à des raisons naturelles. Pierre devient un alter ego de Zola au cours de la longue réflexion philosophique et politique sur les foules, la foi, la religion et la science. Même si, pour créer Pierre, il puise son inspiration auprès d'un prêtre ayant perdu la foi en 1869, Zola "pensait plus probablement encore à lui-même, à ses propres idées» (Mitterand, in E: 476); il «veut un personnage central, celui qui verra et jugera, un autre lui-même» (Ternois, in $\mathrm{E}: 19)$. Telle est l'intention théorique de Zola, qui explique la grande place donnée à la pensée de Pierre.

La thèse que développe le roman comporte trois thèmes.

1. La foi, à laquelle Zola accorde beaucoup de crédibilité, notamment dans le contexte spécifique de son vécu collectif: «La foi déchaînée, la grande force, que rien n'arrêtait plus» (L:389).

2. La religion, dont les obsèques avaient été décrétées par les Lumières et le rationalisme, qui affichait au seuil du nouveau siècle d'importants signes de renaissance; Zola propose le concept d'une «religion nouvelle», qui passe par «le même rêve exaspéré de fraternité et de justice, l'éternel besoin du bonheur ", que les pèlerins partagent avec «les anarchistes [et] les socialistes extrêmes»; une religion nouvelle qui répondrait au «malaise» du siècle; somme toute, le rêve de «la société future» (L: 574-577).

3. La foule, pacifique, existante sous la forme d'une «organisation puissante» (L: 140), contrôlée de façon invisible, une foule où se mélangent toutes les classes.

La foi et le miracle sont des thèmes secondaires de notre article, mais nous nous devons de souligner le fait que le «thème du miracle trouve une surprenante acceptation de la part du "rationaliste" Zola». 
Lourdes «occupe un espace nécessaire» dans l'ensemble de la seconde moitié des œuvres de Zola, lesquelles gravitent toutes «autour de la résurrection ${ }^{30}$ ». Il serait tout à fait intéressant d'établir une comparaison entre la "religion nouvelle », proposée à la fin du roman par le biais de Pierre, avec la «religion d'humanité», proposée par Auguste Comte, ou encore avec le concept de «religions profanes" propres à la société de masse depuis Robespierre ${ }^{31}$. Ici nous signalons, une fois de plus, l'absence de frontières entre le roman et l'essai en ce qui concerne la présentation de thèses. Pour une sociologie de la religion, il serait également intéressant d'analyser le fait que Zola attribue le succès de Lourdes à l'échec de la science:

Ab! tristes hommes, pauvre humanité malade, affamée d'illusion, qui, dans la lassitude de ce siècle finissant, éperdue et meurtrie d'avoir acquis goulûment trop de science, se croit abandonnée des médecins de l'âme et du corps, en grand danger de succomber au mal incurable, et retourne en arrière, et demande le miracle de sa guérison aux Lourdes mystiques d'un passé mort à jamais! (L: 578.)

Concentrons-nous sur la foule de Lourdes. Dès le début, Zola met en exergue «le flot de foule» qui converge à Lourdes: «Ce n'était pas la France seule qui se mettait en marche, mais l'Europe entière, le monde entier.» (L: 49.) D'un point de vue spatial, la foule de Lourdes est différente des concentrations religieuses précédentes, qui avaient généralement lieu dans des espaces fermés telles les cathédrales médiévales. Ici, la foule ne tient pas dans des espaces fermés. C'est une foule de rue, mais cette ressemblance ne fait qu'accentuer les différences qui la distinguent des foules insolentes des villes.

Au cours du séjour à Lourdes, qui correspond à la plus grande partie du roman, il y a une effervescence collective permanente dans les processions, les messes, les parcours, les rencontres, les repas, les hôpitaux, etc. En mesurant la récurrence du terme «foule» dans le roman, on remarque qu'il est constamment présent (168 fois, alors que «multitude» apparaît deux fois), en moyenne une fois toutes les trois pages (Tab. 1). Zola emploie encore d'innombrables fois le terme «cohue», que nous interprétons préférentiellement comme

30. Jean Borie, Zola et les mythes (1971), cité par Naomi Schor, Zola's crowds, Baltimore-Londres, The John Hopkins University Press, 1978, p. 44.

31. Sur ce dernier aspect, voir S. Moscovici, op. cit., p. 461-493. 
foule confuse ou confusion de gens, mais aussi comme synonyme de foule, comme dans «cohue des misérables» (L: 568) et d'autres substantifs collectifs et expressions tels que "peuple(s)», «vague», "vaste mer», «fourmilière», "mer humaine», «océan», "peuple de fidèles», "petit peuple», «masse» et «masses des humbles».

Tableau 1. Occurrences des termes «foule(s)» et «multitude(s)» chez Zola et Huysmans.

\begin{tabular}{lccc}
\hline \multicolumn{1}{c}{ CEuvre } & Ocurrences & Par chapitre & Par page \\
\hline Lourdes, Zola (1894) & 170 & 6,84 & 0,31 \\
Les foules de Lourdes, Huysmans (1906) & 69 & 4,60 & 0,32 \\
\hline
\end{tabular}

Les caractéristiques négatives de la foule, si présentes dans les «foules criminelles » de Germinal, sont mises entre parenthèses dans Lourdes, et cela malgré «la contagion » qui s'empare d'elle, « un délire [...] abolissant les volontés, ne faisant plus de tous ces êtres qu'un être, exaspéré d'amour» (L: 179). La «bête» foule (L: 275) disparaît, la foule de haine et de violence est remplacée par une foule qui vit une «épidémie heureuse, à l'espoir contagieux» de la guérison (L: 225). Zola se laisse impressionner par «la foule, la foule immense» (L: 371), «un troupeau docile» (L: 372, 570), une foule «d'innocence» $(\mathrm{L}: 372,391)$ qui se déplace de façon ordonnée, attirée par quelque chose d'«instinctif, [d']invincible» (L: 371).

La quantité est écrasante et se mue en qualité: "C'était, pendant des heures, le même flot ininterrompu de femmes, d'hommes, d'enfants.» Et «le défilé continuait, continuait sans fin, pendant des jours, pendant des mois, depuis des années; et il semblait que toute la terre vint passer là, au fond de ce coin de rocher, toutes les misères et toutes les souffrances humaines à la file, dans cette sorte de ronde hypnotisée et contagieuse, en quête du bonheur.» (L: 373.) C'est une foule qui à la fois attire comme un aimant, perturbe et répugne:

Pierre n'avait jamais eu une sensation si anxieuse de la foule. Elle était sans menace, d'une innocence et d'une passivité de troupeau; mais il y trouvait un frisson troublant, un souffle particulier qui le bouleversait. Et, malgré son amour des humbles, la laideur des visages, les faces communes et suantes, les haleines gâtées, les vieux vêtements sentant le pauvre, le faisaient souffrir jusqu'à la nausée (L: 391). 
Cette citation laisse aussi transparaître la nouvelle relation ambivalente de l'élite vis-à-vis de la foule: elle sent mauvais, mais le membre de l'élite se déplace parmi elle, l'accepte, se mélange à elle.

On retrouve donc, face à la foule de Lourdes, la double attitude de Zola déjà présente dans Germinal. D’une part, «peur et répugnance», comme la définit Schor, lorsqu'il évoque la comparaison des odeurs corporelles exhalées aussi bien par les pauvres que par les pèlerins malades ${ }^{32}$. D'autre part, Zola est attiré par la force des masses dans la transformation de l'histoire, idée renforcée par la suite dans Fécondité (1899), le premier roman de la série des Quatre évangiles, ouvrage où le pouvoir de la multitude est mis en évidence: "Aucun pas en avant dans l'histoire n'a été fait sans que ce soit le nombre qui pousse l'humanité dans sa marche. Demain, comme ce fut le cas hier, sera conquis par le pullulement des foules en quête de bonheur ${ }^{33}$ ".

Chez Zola et Huysmans, nous retrouvons les deux attitudes de l'élite vis-à-vis de la foule: d'une part, la nouvelle immersion dans ses entrailles; d'autre part, l'ancienne attitude du voyeur, de celui qui contemple la foule de loin. Dans ce dernier registre, on voit que dans Lourdes les manifestations de la foule sont comme un «spectacle» auquel on peut assister de l'extérieur: depuis un pont (L: 280), en tant que «simple spectateur» (L:373-374) ou en observant, depuis une véranda naturelle, «tout un peuple à vol d'oiseau, une fourmilière» (L: 414). On trouvait déjà dans Germinal cette position voyeuriste vis-à-vis de la foule: lorsqu'un groupe de bourgeois assiste depuis les hauteurs, en tant que spectateurs, à la manifestation des mineurs; lorsqu'un groupe de bourgeois suit, à travers les fentes d'un grenier, le passage des manifestants ouvriers («C'était la vision rouge de la révolution qui les emporterait tous, fatalement, par une soirée sanglante de cette fin de siècle»); lorsque le cadavre de Maigrat est castré par la «foule criminelle», tandis que les bourgeoises et la veuve assistent à la scène depuis les fenêtres de la maison ( $G: 410,430)$. Mais il n'y a pas dans Germinal des membres des élites mélangés à la foule. Huysmans fait également référence au «spectacle auquel on assiste, du bord de cette terrasse» sur «l'immense cirque de l'esplanade», «une masse compacte, le public», « un unique troupeau, abrité sous une seule et même tente» $(\mathrm{H}: 159,149,160)$. Mais lui aussi

32. N. Schor, op. cit., p. 127.

33. Cité ibid., p. 119. 
partage l'autre nouvelle attitude élitiste: "Je me mêle à la foule.» $(\mathrm{H}: 177$.

Les aspects folkloriques des habits, les odeurs et les comportements de la foule des malades, de riches et de pauvres ou de fidèles en transe corroborent la thèse de Zola sur la foule seulement dans la mesure où, malgré leur caractère négatif, ils ne s'opposent pas à un changement de comportement des personnages élitistes et donc de l'auteur lui-même. Il passe dans Germinal d'une attitude de peur de la cohue, mais aussi de sympathie et de compréhension vis-à-vis de la révolte des mineurs, à une attitude d'immersion dans Lourdes.

Toutefois, ceci ne constitue pas l'essentiel de la nouvelle position de Zola sur le sujet. Si le père Pierre se propose bel et bien de lutter pour une «religion nouvelle», ce roman expose, pour la première fois nous semble-t-il, les fondements d'une foule nouvelle, dont Zola, le journaliste-ethnologue-homme de fiction, est le découvreur.

Le nombre de pèlerins et l'intensité de la foi produisent, dans ce roman, un effet remarquable et sans doute inespéré chez Zola: c'est la foule qui guérit, qui fait des miracles. La foule de Lourdes agit de façon positive. Son action fait que, par la foi, les croyants croient en la guérison. Zola met en avant cette conception qui, selon lui, n'est pas encore étudiée par la science (médecine, psychologie, sociologie):

Pierre, alors, commença à comprendre ce qui se passait à Lourdes, l'extraordinaire spectacle auquel le monde assistait depuis des années, parmi l'adoration dévote des uns et la risée insultante des autres. Évidemment, des forces mal étudiées encore, ignorées même, agissaient: autosuggestion, ébranlement préparé de longue main, entraînement du voyage, des prières et des cantiques, exaltation croissante; et surtout le souffle guérisseur, la puissance inconnue qui se dégageait des foules, dans la crise aiguë de la foi. [...] Et, de tout cela, sortait, évidente, l'impossibilité de prouver que le miracle était ou n'était pas. Dès ce moment, le miracle ne devenait-il pas une réalité, pour le plus grand nombre, pour tous ceux qui souffraient et qui avaient besoin d'espoir? (L: 214, nous soulignons.)

Il s'agit d'un tour de force de l'argumentation. Zola explique «scientifiquement» les guérisons comme une intervention de la foule «dans la crise aiguë de la foi ». Cette thèse du roman sera reprise bien plus tard par la sociologie: dans Collective behavior (1992), Erich 
Goode soutient que les dynamiques de la foule «valident les miracles que les personnes disent avoir lieu ${ }^{34}{ }^{\prime}$. Dans Lourdes, la religion apparaît comme l'expression de la société elle-même - dans ce cas précis, sous la forme de la foule -, une notion qui sera développée par Durkheim, pour qui «Dieu est la société ${ }^{35}$ ». Non seulement Zola attribue les guérisons de Lourdes à la foule, mais il ajoute qu'il est insignifiant de prouver le miracle: il s'agit d'une œuvre collective qui se transforme en croyance collective. Cette hypothèse, ou thèse en forme de fiction, était novatrice et prolongeait celle que Charcot avait publiée en 1892, l'année même où Zola enquêtait à Lourdes (et que Zola a lu-Ternois, in $\mathrm{E}: 19)$. Charcot affirmait que les guérisons miraculeuses n'avaient lieu que dans les cas de manifestations physiques de troubles mentaux: "Ces tumeurs et ces ulcères autour desquels on mène tant de bruit sont aussi de nature hystérique» tout comme le "faith-healing des convulsions et des paralysies ${ }^{36}$ ".

La science et le déterminisme de Charcot et de Zola s'attaquaient à des phénomènes inexpliqués. En 1899, le médecin portugais José de Lacerda inscrivait le phénomène de Lourdes «dans la lutte entre les sciences et la religion». Il ne niait aucunement les guérisons de Lourdes, mais, s'appuyant sur des analyses chimiques, il contestait toute propriété guérisseuse de son eau. Dans le sillage de Zola, il affirmait que les guérisons attribuées à l'eau étaient dues à l'action de la foule: cette eau est «supérieurement efficace dans sa propre source, lors de grandes occasions solennelles, lorsque les malades, en foules de pèlerins, dans une réciproque et violente contagion par la foi, par le biais d'ardentes prières et de chants sacrés, vont demander la santé à l'eau miraculeuse.» L'action de la foule devient alors faith healing. Lacerda considérait donc qu'il s'agissait d'un phénomène de «foi violente» et d'une «hallucination» déclenchée par Bernadette, cette "géniale hallucinée». Lorsqu'elle «se montrait aux pèlerins, devant la Grotte, un vent fort de foi et de folie, agitait les foules, faisant éclater un long bourdonnement de sanglots et de prières ${ }^{37}$ ». Ainsi, à l'époque, l'idée exprimée par Zola du miracle en tant qu'œuvre collective qui se trans-

34. Cité par Ph. Davis, J. Boles, art. cit., p. 375.

35. Émile Durkheim, Textes 2, Religion, morale, anomie, Paris, Éd. de Minuit, 1975, p. 10.

36. Jean-Martin Charcot, «La foi qui guérit», la Revue hebdomadaire, 1892, voir www.psychanalyse-paris.com/La-foi-qui-guerit (consulté le 5/9/2010).

37. José de Lacerda, «Milagres», Medicina contemporânea, XVII, 11, 12 mars 1899; Id., «Milagres (2)», ibid., XVII, 12, 19 mars 1899. Nous soulignons. 
forme en croyance a eu un certain impact. Zola la présente dans un contexte quasi scientifique sous forme d'une «enquête» de Pierre qui, à bien voir, anticipait celle des hommes de sciences de son temps:

Peu à peu, malgré son effort, il retournait à son enquête, il doutait, il cherchait. Ainsi, quelle était donc la force inconnue qui se dégageait de cette foule, un fluide vital assez puissant pour déterminer les quelques guérisons qui, réellement, se produisaient? Il y avait là un phénomène qu'aucun savant physiologiste n'avait encore étudié. Fallait-il croire qu'une foule n'était plus qu'un être, pouvant décupler sur lui-même la puissance de l'autosuggestion? Pouvait-on admettre que, dans certaines circonstances d'exaltation extrême, une foule devînt un agent de souveraine volonté, forçant la matière à obéir? Cela aurait expliqué comment les coups de guérison subite frappaient, au sein même de la foule, les sujets les plus sincèrement exaltés. Tous les souffles se réunissaient en un souffle, et la force qui agissait était une force de consolation, d'espoir et de vie (L: 395-396, nous soulignons).

La foule comme «agent de souveraine volonté »: voici une thèse qu'aucun théoricien de ce champ n'avait encore osé appliquer à une foule positive. L'idée était déjà exprimée ici et là dans les thèses sur les «foules criminelles» car, ne faisant qu'un seul être, la cohue avait une vie indépendante de celle de ses membres, ce qui permit aux criminologues et juristes de créer la notion de «semi-responsabilité » dans les crimes collectifs (de manière à absoudre ou à diminuer les peines des membres des foules lors des jugements) ${ }^{38}$.

La réflexion de Lourdes sur la foule contient un autre élément d'analyse intéressant, qui sera repris, dès l'année suivante, lorsque Le Bon publie sa Psychologie des foules. Il s'agit du rêve de bonheur, qui est essentiel dans la caractérisation de cette foule: «Un cri d'exécration montait du petit peuple, de tous les déshérités qui avaient besoin de merveilleux autant que de pain, pour vivre» ( $\mathrm{L}: 235$, nous soulignons). Par cette catégorie du «merveilleux» placée sur le même plan que le besoin de pain (le cirque, dans les vers panem et circenses, de Juvénal), Zola attribue au rêve un rôle moteur dans la mise en

38. Sighele affirme que Giuseppe Pugliese, dont il a subi l'influence, a été le premier à exposer, dans Del delitto collettivo (1887), «la doctrine de la responsabilité pénale dans le crime collectif» (Scipio Sighele, La foule criminelle. Essai de psychologie criminelle [ $1^{\text {re }}$ éd. en it. $1891 ; 1^{\text {re }}$ éd. en fr. 1892], Paris, Félix Alcan, 1901, p. 29). 
mouvement de la foule. On peut ainsi déduire que celui qui apporte le rêve à la foule (sous la forme du religieux, du politique) devient de ce fait capable de la contrôler. Il s'agit d'un aspect fondamental: il est possible de diriger la foule, de la modeler. L'abbé Peyramale, à l'origine du culte à Lourdes, était un "conducteur d'hommes", un «remueur de foules» et, avant lui, Bernadette elle-même - si elle l'avait voulu - aurait pu diriger la foule et créer sa propre religion (L:338).

De la même façon que l'immense foule amoureuse permet à Pierre de créer l'idéologie de la «religion nouvelle», imprégnée d'idées de justice, Zola formule une proposition politique opposée, par le biais du personnage de Berthaud, un conservateur qui souhaiterait le retour de la monarchie catholique: pour lui, «Lourdes était surtout un foyer de propagande, où il contentait ses rancunes politiques, se réjouissait des pèlerinages nombreux, qu'il croyait être désagréables au gouvernement. Ah! Si l'on avait pu amener les ouvriers des villes, créer une démocratie catholique!» (L: 414-415.) Berthaud établit la différence entre les foules criminelles et amoureuses: «C'est une leçon donnée à leur République. On se tue, à Paris, quand des foules pareilles célèbrent quelque date sanglante de leur exécrable histoire... Qu'ils viennent donc ici s'instruire!» Cet homme de droite est à l'aise au milieu de la foule: "Il n'était jamais si heureux, à Lourdes, qu'au milieu des grandes affluences de fidèles.» (L: 512.) Qui aurait osé imaginer, avant ce roman, un conservateur heureux au milieu de la foule?

En résumé, il y a dans ce roman trois conceptions de la foule.

1. La «foule criminelle», à laquelle Zola n'accorde pas d'importance, qui apparaît tout juste en contrepoint.

2. la foule d'une nouvelle "religion de la souffrance humaine» et du rêve, par la fraternité et la justice, de la quête du bonheur pour les pauvres et les souffrants, pour qui la science, compte tenu de son incapacité, devrait être forcée à «laisser une porte ouverte sur le mystère» (L: 571-577).

3. la foule d'un «nouveau peuple» (L: 512), sous la houlette de la droite au service d'une politique qui pourrait être contraire aux intérêts matériels (mais non spirituels) de ce même peuple.

Comme nous y avons déjà fait allusion, cette dernière est précisément la proposition implicite que contient le petit livre de Le Bon sur la Psychologie des foules. Et la foule pacifique, comme nous l'avons également vu, devient une nouvelle façon de concevoir la 
foule, comme le note Gabriel Tarde en 1898. C'est pourquoi il nous semble indispensable de signaler la portée de Lourdes, un roman à thèse, dans le passage de la théorisation française de la foule criminelle vers une foule façonnable par ses meneurs.

\section{Joris-Karl Huysmans.}

\section{La méprisable, nécessaire foule contrôlée par les institutions}

1906: douze ans après Lourdes, trois ans après la mort de Zola, le romancier français d'origine hollandaise Joris-Karl Huysmans publie un essai sous forme de récit: Les foules de Lourdes. Le titre est un pléonasme, comme le remarque Schor: «En fait, dans la seconde moitié du XIX ${ }^{\mathrm{e}}$ siècle, Lourdes et foules sont des synonymes ${ }^{39}$.» Bien que ce soit un récit et non une fiction, cet ouvrage est, pour notre optique, moins digne d'intérêt que celui de Zola, non seulement parce qu'il lui succède et en reproduit certaines idées, mais aussi à cause de sa propre intention théorique, domaine où Zola a innové alors que Huysmans ne fait que suivre.

Huysmans a visité Lourdes en 1903 et 1904. Il partage avec Zola certaines idées et conceptions : il souligne la «communion des âmes » dans la foule de Lourdes; il accepte le mélange de l'élite avec la foule pauvre, même si, simultanément, il préfère adopter l'attitude de celui qui la regarde du dehors et comme un spectacle. Observons d'autres ressemblances et, surtout, ce qui le sépare de Zola.

D'un point de vue formel, son livre se distingue de celui de Zola parce que c'est un récit personnel, descriptif et chargé d'opinions. Toutefois, cet aspect est sans importance pour ce qui nous intéresse, car le naturalisme de Zola le rapproche de la «transparence» journalistique et de la spéculation scientifique de son époque. Zola luimême donne son opinion par le biais de ses personnages.

Les points de vue diffèrent: Zola défend une nouvelle conception de la foule "innocente» et qui se conduit convenablement. Il comprend la foi, mais il rejette toute intervention divine dans les guérisons. Huysmans partage la même «théorie» nouvelle sur la foule,

39. N. Schor, op. cit., p. 126. Initialement, l'ouvrage de Huysmans devait s'intituler Les deux faces de Lourdes, parce qu'il y avait, à côté du «Lourdes du Bon Dieu: lieu qui draine le flux des foules médiévales pénitentes», le "Lourdes satanisé: cuvette maléficiée, le bidet-satan où s'accumule la lie de toutes les délavures diaboliques, au fond baptismal répond la cuvette bordel», comme l'écrit François Angelier, en reprenant l'argumentation de l'auteur (in $\mathrm{H}: 27-28$ ). 
mais, converti au catholicisme depuis 1892, il la met au service exclusif du catholicisme et défend l'intervention divine, miraculeuse.

Contrairement à Zola, chez qui on retrouve des émotions qu'il partage avec les foules décrites, Huysmans les déteste: «je n'aime pas les foules qui processionnent, en bramant des cantiques» et qui prennent «d'assaut» l'espace public de Lourdes. Toutefois, il se dit prêt à endurer le sacrifice: "Je me mêle à la foule.» (H: 177.) Il partage le point de vue cinglant de Grillot de Givry dans Lourdes, ville initiatique (1902):

Toute foule est méprisable par elle-même; c'est un vain tourbillon qui se vautre dans l'ignoble et se perd dans la mort; et cependant la foi est la régénératrice des foules et peut les hausser au niveau d'une personnalité. La foule éprise du mystère émet un courant de perfection qui, s'emparant de chacun de ses membres, les unit en un même espoir et une même croyance: alors ainsi transformée, elle offre le spectacle d'un ordre et d'une harmonie parfaite (cité par $\mathrm{H}: 44$, nous soulignons).

Huysmans fait aussi référence à la foule en termes de "cohue», «horde», «meute», «amas», «tourbe», «flux». La foule est «bruyante», affectée par sa «promiscuité» $(\mathrm{H}: 120,129)$ et sa « démence», ce qui le conduit à se retirer et à l'observer de loin. Mais cette foule est, véritablement, l'essence du phénomène. Huysmans fait honneur au titre qu'il a choisi: le terme «foule(s)» se répète cinquante et une fois et le terme «multitude(s)» dix-huit. Dans l'ensemble, on les retrouve aussi souvent que dans le roman de Zola: soit, toutes les trois pages (Tab. 1).

Pour Huysmans, la foule de Lourdes est détestable mais nécessaire, pacifique et ayant pour seule utilité celle de servir la religion. Lui aussi, à l'instar de Zola, s'étonne de sa bonté et de sa discipline, au point d'utiliser la même expression que Givry: «Ce qui est vraiment extraordinaire [...] dans cette promiscuité continue de gens de tous les pays dont beaucoup ne comprennent même pas le français, c'est une discipline amicale et un ordre parfait.» (H: 99, nous soulignons.)

Cette caractéristique, qui se fond avec la liminalité de la période de pèlerinage, nous permet de progresser vers un thème déjà mis en avant par Zola: la fusion de tout type de personnes dans la foule de Lourdes. Tandis que les foules urbaines ou rurales étaient uniquement formées par les éléments d'un groupe social donné (agriculteurs, tra- 
vailleurs ruraux, ouvriers), dans la foule unie par la foi se rejoignent riches et pauvres. La «communion des âmes", évoquée à trois reprises (H: 84-85, 187), permet que se «confondent les classes» $(\mathrm{H}: 85)$, qu'il $\mathrm{y}$ ait «fusion des classes» $(\mathrm{H}: 261)$ : «Lourdes est, en somme, une principauté qui réalise, et bien au-delà, les plus audacieuses chimères des philanthropes; c'est la fusion temporaire des castes» ( $\mathrm{H}: 183)$.

Plus qu'avec Zola, le «Lourdes» de Huysmans revêt un caractère politique, même s'il ne l'exprime pas en ces termes. Sa position est ambivalente, mais elle participe clairement d'une situation qui semblait n'avoir que deux portes de sortie: «Il n'y avait pour l'idéologie sous-jacente à l'esthétique naturaliste que deux voies possibles: le socialisme ou le mysticisme; c'est pour ce dernier qu'opta J.-K. Huysmans ${ }^{40}$ ». Ce choix supposément apolitique de la religion est essentiel à l'ouvrage de Huysmans, qui présente la foule comme un agrégat «de toutes classes» qui prie et ne forme «qu'un unique troupeau» $(\mathrm{H}: 160)$. Il s'accorde avec la stratégie du catholicisme ultramontain qui comptait sur l'essor des pèlerinages pour «attirer les masses populaires dans son camp et faire de ces manifestations religieuses une opposition au régime constitutionnel ${ }^{41}$ ».

Tandis que Zola voyait Lourdes en partie comme une conséquence de l'échec de la science et du progrès à procurer du bonheur aux gens, Huysmans la voit comme conséquence de l'échec de la démocratie de masse, qui provoque à la fois de l'insolence et du désenchantement face à «l'état morbide d'une société qui a été trop dupée par le mensonge des décors et par l'abus des apparences. [...] Personne ne croit plus à l'honnêteté des hommes politiques, à la valeur des généraux, à l'indépendance des magistrats; personne ne se figure que le clergé est composé de saints"; il y a donc là comme «une sorte de malaria de l'irrespect» vis-à-vis des élites (H:200). Le rapport entre les élites et les masses était un thème à propos duquel on épiloguait sans cesse en France, depuis que le pays avait réintroduit le suffrage universel masculin en 1884. La plupart des observateurs s'étaient rendu compte de la contradiction entre les manœuvres en coulisses de la bourgeoisie financière et intellectuelle, et sa rhétorique publique, destinée à plaire aux masses ${ }^{42}$. Or, suggère Huysmans, Lourdes procure une solution

40. Françoise Gaillard, "Huysmans (1848-1907)», in Encyclopaedia universalis, Corpus XI, 1996, p. 762.

41. Vítor Neto, O Estado, a Igreja e a sociedade em Portugal (1832-1911), Lisbonne, INMC, 1998, p. 464.

42. J. Van Ginneken, op. cit., p. 150. 
au désenchantement et à l'insolence des masses. Lourdes est née d'une «nécessité» de reconduire les pauvres au sein du troupeau: «Dieu ne choisit que les plus pauvres et les plus humbles [dans ce cas précis Bernadette], lorsqu'il a besoin d'un truchement pour s'adresser aux masses.» (H: 221.) La «Vierge a voulu des foules, ainsi qu'au Moyen Âge. Elle les a.» (H:259.) Même si Huysmans situe Lourdes $\mathrm{au}$ «temps des grands pèlerinages internationaux» $(\mathrm{H}: 87)$, il atténue la nouveauté du phénomène en identifiant ces foules avec celles du Moyen Âge. C'est précisément l'inverse de ce qu'avait écrit Zola en 1894: "Jamais plus la nation entière ne se prosternerait, comme l'ancienne nation croyante, dans les cathédrales du XII ${ }^{\mathrm{e}}$, pareille à un troupeau docile sous les mains du Maître.» (L: 570.) Ainsi, Zola voyait Lourdes comme une réponse du catholicisme à la dérive des croyants, tandis que pour Huysmans les foules «sont les mêmes" que celles du Moyen Âge: «L'âme ingénue et la foi naïve des vieilles paysannes n'ont guère changé; l'existence même que ces multitudes mènent, ici, [...] rappelle la vie des cohues d'antan, couchant dans la cathédrale de Chartres.» (H:259.)

On peut affirmer que Huysmans a voulu contredire Zola ${ }^{43}$. Il formule une dizaine de critiques ou de démentis à son roman, notamment en ce qui concerne la preuve des miracles: pour lui, Zola est «résolument inexact» $(\mathrm{H}: 116$; voir aussi: 215-221, 240, 250, 264-265). Ses critiques, autrement acerbes que celles de Zola visà-vis de certains aspects de Lourdes, s'expliquent par son élitisme très prononcé. Non seulement Huysmans abomine les foules, mais il condamne aussi, plus âprement que Zola, l'horreur artistique du sanctuaire qu'il considère tout juste nécessaire en tant que preuve de la présence du diable jusque dans un lieu sacré (L: 125-126). À propos de la thématique qui nous intéresse, Huysmans prétend démentir Zola en ce qui concerne les facultés extraordinaires de la foule: il le cite pour nier que de la «puissance inconnue des foules» émane un «souffle guérisseur »: «Cette puissance dont le vrai nom est la prière est indéniable, mais, je le répète, elle n'est pas indispensable au salut des malades, pas plus, d'ailleurs que le cadre et le milieu» (H:265). Pour Huysmans, tout s'explique par la foi et relève du religieux.

43. Huysmans ne s'est pas illustré dans le roman naturaliste comme Zola. Leurs carrières ont suivi des chemins opposés. Ils ne fréquentent plus le même cercle littéraire dès 1892, année de la conversion de Huysmans, qui a surpris Zola; c'est d'ailleurs par la presse que Huysmans prend connaissance du projet de Zola d'écrire un roman sur Lourdes (gallica.bnf.fr/Zola/RecepAdap/notesGuermes.htm). 


\section{La foule religieuse, expression du culte}

Souligner le rôle de la foule dans la religion comme l'ont fait Zola et Huysmans, c'est aussi, comme le fait Durkheim en 1912, remarquer «le rôle prépondérant du culte dans toutes les religions, quelle qu'elles soient ${ }^{44} »$. Ce que Zola analysait par le biais de la fiction, ce qui était le prolongement de cette réflexion chez Huysmans - même si c'était pour la contredire - est devenu de la science sociologique dans Les formes élémentaires de la vie religieuse. En s'attardant sur le rôle du culte, Durkheim conclut:

La société ne peut faire sentir son influence que si elle est un acte, et elle n'est un acte que si les individus qui la composent sont assemblés et agissent en commun. C'est par l'action commune qu'elle prend conscience de soi et se pose; elle est avant tout une coopération active. Même les idées et les sentiments collectifs ne sont possibles que grâce à des mouvements extérieurs qui les symbolisent [...]. C'est donc l'action qui domine la vie religieuse par cela seul que c'est la société qui en est la source ${ }^{45}$.

En étudiant Lourdes comme journaliste-ethnologue et homme de fiction, Zola anticipait la conclusion de Durkheim que voici: les hommes «sentent [...] que la vraie fonction de la religion n'est pas de nous faire penser, d'enrichir notre connaissance, d'ajouter aux représentations que nous devons à la science des représentations d'une autre origine et d'un autre caractère, mais de nous faire agir, de nous aider à vivre ${ }^{46}$ ». La foule sublime, donc, et l'action en pensée religieuse par le biais du culte. Et, comme Zola, Durkheim perçoit dans la communion-foule la possibilité pour l'individu de se convaincre que le bonheur est possible: «Le fidèle qui a communié avec son dieu n'est pas seulement un homme qui voit des vérités nouvelles que l'incroyant ignore; c'est un homme qui peut davantage. Il sent en lui plus de force soit pour supporter les difficultés de l'existence soit pour les vaincre ${ }^{47}$.»

44. Émile Durkheim, Les formes élémentaires de la vie religieuse. Le système totémique en Australie (1912), Paris, Puf, 1990, p. 598.

45. Ibidem.

46. Ibid., p. 595.

47. Ibidem. 


\section{Conclusion}

Nous avons analysé l'articulation entre le phénomène religieux de Lourdes et la théorie sociopolitique de la foule, à partir de deux œuvres littéraires de référence (Zola et Huysmans). Nous avons vérifié que Zola propose la thèse d'une foule qui est à l'origine de guérisons miraculeuses, à travers l'exacerbation de la foi des croyants, et présente deux positions alternatives sur cette foule religieuse: ou comme support d'une «religion nouvelle» (position dont il serait le tenant) ou comme support d'un système politique conservateur. Nous avons vu cette deuxième position assumée par Huysmans.

Nous avons aussi vérifié que Zola a été le premier à développer une analyse et une conception de la foule religieuse d'un point de vue sociopolitique, avant Sighele, Tarde, Le Bon et, bien sûr, Durkheim, priorité qui jusqu'à présent n'a pas été signalée par les travaux sur l'histoire de l'étude de la foule. En fait, contrairement à Germinal, roman sur la foule ouvrière, voire "criminelle», Lourdes n'a jamais été considéré comme un jalon de cette histoire. Ni le roman, ni sa conception originale ne font l'objet d'une quelconque étude de la part de Barrows, Ginneken et Moscovici, ni même dans l'œuvre que Schor a consacrée aux foules de Zola et dans laquelle elle considère pourtant Lourdes comme «une des plus importantes fictions-foule de Zola ${ }^{48}$ ".

Le fait que Lourdes soit une œuvre de fiction est accessoire. Tout d'abord, parce que tous les spécialistes de la foule depuis Taine ont été influencés ou se sont inspirés de récits littéraires pour leurs analyses scientifiques. Ensuite, parce que Zola, en tant que journalisteethnologue, «sociologue praticien» ou encore auteur naturaliste et de romans à thèse, était, sous les traits du «romancier expérimental», un passeur d'observations et d'analyses pour ses contemporains. Il était aussi un propulseur de théorie qui ne peut être mis de côté, de la même façon que l'on ne rejette pas Sighele ou Tarde lorsqu'ils s'appuient sur le même Zola ou sur de nombreux autres romanciers ayant dépeint des foules tout au long du $\mathrm{XIX}^{\mathrm{e}}$ et au début du $\mathrm{XX}^{\mathrm{e}}$ siècle.

\section{Traduit du portugais par Manuel Antunes da Cunba *}

48. N. Schor, op. cit., p. 126.

* La traduction a été financée par le Centre d'études de la communication et de la culture de la faculté de sciences humaines de l'Université catholique portugaise (CECC-UCP). 